\title{
Clinical feasibility of using effective atomic number maps derived from non-contrast spectral computed tomography to identify non- calcified atherosclerotic plaques: a preliminary study
}

\author{
Wenping Chen ${ }^{1 \#} \wedge$, Ran Li $^{2 \#}$, Kejie Yin ${ }^{1 \#}$, Jing Liang ${ }^{1}$, Hui Li ${ }^{1}$, Xingbiao Chen ${ }^{3}$, Zhihong Sheng ${ }^{3}$, \\ Hongming $\mathrm{Yu}^{1}$, Dan $\mathrm{Mu}^{1}$ \\ ${ }^{1}$ Department of Radiology, Nanjing Drum Tower Hospital, The Affiliated Hospital of Nanjing University Medical School, Nanjing, China; \\ ${ }^{2}$ Department of Cardiology, Nanjing Drum Tower Hospital, The Affiliated Hospital of Nanjing University Medical School, Nanjing, China; ${ }^{3}$ Clinical \\ Science, Philips Healthcare, Shanghai, China
}

Contributions: (I) Conception and design: D Mu, H Yu; (II) Administrative support: D Mu; (III) Provision of study materials or patients: J Liang, H Li; (IV) Collection and assembly of data: R Li, K Yin, Z Sheng; (V) Data analysis and interpretation: W Chen, R Li, K Yin, X Chen; (VI) Manuscript writing: All authors; (VII) Final approval of manuscript: All authors.

\#These authors contributed equally to this work.

Correspondence to: Hongming Yu; Dan Mu. Department of Radiology, Nanjing Drum Tower Hospital, The Affiliated Hospital of Nanjing University Medical School, 321 Zhongshan Road, Nanjing 210008, China. Email: yhm239x@126.com; mudan118@126.com.

Background: To assess the clinical feasibility of using effective atomic number (Zeff) maps derived from non-contrast-enhanced computed tomography (NCECT) scans obtained by dual-layer spectral computed tomography (DLCT) to identify non-calcified atherosclerotic plaques.

Methods: A total of 37 patients with 86 non-calcified atherosclerotic plaques confirmed by contrast-enhanced CT (CECT) were enrolled in this retrospective study. Both spectral-based-images (SBI) and conventional images (CI) were reconstructed from NCECT and CECT scans. The presence of plaques on NCECT Zeff maps and CIs were independently assessed by 2 radiologists. In CECT scans, plaques and regions of interest (ROIs) in vessel lumens were assessed with CT attenuation and Zeff values, and the proportion of plaques was determined as Area (plaque)/Area (vessel). The CT and Zeff values for plaques and blood were recorded from both CECT and NCECT scans. Contrast-to-noise ratios (CNRs) of the plaques were calculated and compared using CT attenuation and Zeff values. Finally, interobserver agreement was evaluated.

Results: A total of 47 of the $86(54.7 \%)$ plaques were identified on Zeff map images derived from the NCECT scans while only 7 (8.1\%) plaques were identified on the CI. There was no significant difference between the mean vessel ROI area measured on CIs and that measured on Zeff map images (502.19 vs. $\left.498.14 \mathrm{~mm}^{2} ; \mathrm{P}=0.28\right)$, while the mean plaque ROI area was larger $\left(81.45\right.$ vs. $\left.75.46 \mathrm{~mm}^{2}\right)$. The observer consensus of vessel and plaque ROI area measurements using both methods was excellent, with interclass correlation coefficients (ICCs) of 0.99 and 0.94, respectively. For the 7 plaques detected both by NCECT CI and Zeff mapping, the CT attenuation and Zeff blood values were both larger than the plaque values [42.00 vs. 25.67 Hounsfield unit (HU); 7.33 vs. $7.19 \mathrm{HU}$; both $\mathrm{P}<0.05]$; the plaque ROI area measurement on the NCE Zeff map was smaller than that on the CE CI (48.73 vs. $\left.77.76 \mathrm{~mm}^{2}\right)$, but was much larger than that on the NCE CI $\left(18.39 \mathrm{~mm}^{2}\right)$. For all 47 plaques detected by NCE Zeff mapping, the CT attenuation and Zeff values of blood and plaques on the NCECT images showed no significant differences (42.53 vs. $35.14 \mathrm{HU}$; P=0.18;

\footnotetext{
$\wedge$ ORCID: 0000-0001-5309-9735.
} 
7.32 vs. 7.31, $\mathrm{P}=0.71)$; however, the $\mathrm{CNR}$ of Zeff was significantly higher than the CT attenuation value (1.69 vs. $1.12 ; \mathrm{P}<0.05$ ) derived from the NCECT scans. Inter-reviewer agreement was good (ICC $=0.78$ ).

Conclusions: Zeff map images derived from NCECT SBI with DLCT provide a potentially feasible approach for identifying non-calcified atherosclerotic plaques, which might be clinically useful for the screening of asymptomatic at-risk patients.

Keywords: Atherosclerosis; non-calcified plaque; dual-energy CT; effective atomic number.

Submitted Jun 17, 2021. Accepted for publication Dec 14, 2021; Published online: 13 Jan 2022.

doi: 10.21037/qims-21-643

View this article at: https://dx.doi.org/10.21037/qims-21-643

\section{Introduction}

Atherosclerosis is a systemic disease that progresses slowly and silently over decades (1). The long-term disease course provides an opportunity for diagnosis before symptoms occur. Early detection and prevention of adverse events is crucial in clinical practice. Contrast-enhanced (CE) computed tomography (CT), a non-invasive method for detecting atherosclerotic disease, has played an increasingly important role in cardiovascular imaging modalities in recent years $(2,3)$. However, CECT requires the injection of contrast agents, which is not recommended for patients with severely reduced kidney function or for the regular screening of atherosclerotic burden in routine health examinations.

Dual-energy CT (DECT), an emerging technology developed in the last decade, allows for material decomposition by absorbing high- and low-energy X-rays $(4,5)$. Compared with conventional CT, DECT provides more image types, such as iodine maps, effective atomic number (Zeff) maps, and virtual mono-energy images, which can yield more useful diagnostic information in some clinical areas $(6,7)$. Dual-layer spectral-detector CT (DLCT) is the latest commercially available DECT that acquires high- and low-energy $\mathrm{X}$-rays by using a single $\mathrm{X}$-ray tube and double-layer stacked detectors $(8,9)$. This detectorbased DECT approach provides exactly matched highand low-energy datasets, which enables raw data-based, material-decomposition methods and delivers accurate quantitative measurements for iodine concentrations and Zeff values (10-12). Another important benefit of this approach is the on-demand retrospective dual-energy analysis which does not require dual-energy scan settings beforehand (13). Indeed, the dual-energy mode is always on while scanning with DLCT, with no need for changing the existing scan parameters or clinical workflow. Furthermore, retrospectively generated spectral image series can be helpful for material characterization or lesion detection.

In conventional CT, non-calcified atherosclerotic plaques are hardly identified on non-contrast-enhanced (NCE) $\mathrm{CT}$ images due to the broad CT attenuation value range [3-152 Hounsfield units (HU)] and poor contrast with blood $(17-84 \mathrm{HU})(14,15)$. To the best of our knowledge, only a few studies have detected non-calcified plaques on conventional NCECT images $(16,17)$, and no other study has focused on NCE DECT images. However, the findings of studies assessing DLCT have indicated that Zeff map images could improve the detection of occult lesions or isodense stones, indicating its superiority over conventional CT (18-20).

The purpose of this study was to investigate the clinical feasibility of using Zeff map images derived from NCE scans obtained by DLCT to detect non-calcified atherosclerotic plaques.

We present the following article in accordance with the Materials Design Analysis Reporting (MDAR) checklist (available at https://qims.amegroups.com/article/ view/10.21037/qims-21-643/rc).

\section{Methods}

This retrospective study was approved by the local Ethics Committee of Nanjing Drum Tower Hospital, The Affiliated Hospital of Nanjing University Medical School (project ID: 2021-026-02), and written informed consent was waived. The study was conducted in accordance with the Declaration of Helsinki (as revised in 2013).

\section{Study population}

From June 2019 to August 2019, 285 consecutive patients with suspected thoracic or abdominal diseases underwent CE and NCECT scans, and were retrospectively evaluated 
in this study. The exclusion criteria were as follows: (I) age $<18$ years, (II) no non-calcified plaque confirmed by CECT, (III) imaging data incomplete for analysis, and (IV) substantial image artifacts. Finally, a total of 37 patients with 86 non-calcified atherosclerotic plaques were assessed.

\section{Data acquisition}

Both NCE- and CE-CT scans were performed on a 128-slice DLCT scanner (IQon; Philips Healthcare, Best, The Netherlands) with the following parameters: tube voltage, $120 \mathrm{kVp}$; tube current lower and upper limit, 40 and $250 \mathrm{mAs}$, respectively; collimation, $64 \times 0.625 \mathrm{~mm}$; pitch, 0.9 ; rotation time, $0.5 \mathrm{~s}$; reconstruction field of view, $500 \mathrm{~mm}$; reconstruction matrix, $512 \times 512$; dose modulation, enabled, DoseRight Index =21, (3D-DOM; Philips Healthcare). Spectral-based-images (SBI) and conventional images (CI) were then reconstructed and saved to the dedicated workstation (Intellispace 9; Philips Healthcare). Zeff images were created from SBI retrospectively for both the CE and NCE scans, using the spectral CT viewer from the dedicated workstation.

\section{Plaque analysis}

Plaque analysis was performed independently by 2 radiologists with more than 5 years of experience in radiology. In case of disagreement, a third senior radiologist with more than 17 years of experience in radiology was invited to make the final decision. The 2 radiologists were blinded to the detailed information of images and were asked independently to review the $\mathrm{CE}$ conventional $\left(\mathrm{CE}_{\mathrm{cov}}\right)$, CE Zeff $\left(\mathrm{CE}_{\text {Zeff }}\right)$, NCE conventional $\left(\mathrm{NCE}_{\text {cov }}\right)$, and $\mathrm{NCE}$ Zeff $\left(\mathrm{NCE}_{\text {Zeff }}\right)$ images. The radiologists were allowed to change the window setting manually to enhance the visual detection of plaque. To reduce recollection bias, only 1 image series was provided at a time, with 2 weeks allowed for the review.

A region of interest (ROI) was placed on the plaque or blood vessel using the slice with the largest plaque diameter to measure CT attenuation, Zeff values, and ROI areas. For $\mathrm{CE}_{\text {cor, }} \mathrm{CT}$ attenuation values of the plaque and blood, plaque (Area $\left.a_{\text {plaque }}\right)$, and vessel (Area $\left.a_{\text {vessel }}\right)$ ROI areas were recorded; the plaque proportion was calculated as Area plaquel Area $_{\text {vessel. }}$. For $\mathrm{CE}_{\text {Zeff, }}$ Zeff values of the plaque and blood, the plaque, and vessel ROI areas were recorded; plaque proportion was also calculated. For $\mathrm{NCE}_{\text {cor }}$ CT attenuation values and standard deviations (SD) for the plaque and blood were recorded. For $\mathrm{NCE}_{\text {Zeff }}$ Zeff values and SD for the plaque and blood were recorded. The contrast-tonoise ratio (CNR) of the Zeff or CT attenuation value was calculated using the following equation:

$$
C N R=\left|\frac{\text { Plaque }- \text { Blood }}{S D_{\text {blood }}}\right|
$$

The total number of plaques detected using $\mathrm{NCE}_{\text {cov }}$ and $\mathrm{NCE}_{\text {Zeff }}$ were recorded, respectively. The plaque detection rate using the NCECT scan was defined as:

$$
\text { Detection Rate }=\frac{N_{N C E}}{N_{C E}} \times 100 \%
$$

where $\mathrm{N}_{\mathrm{NCE}}$ is the total number of plaques detected on $\mathrm{NCE}_{\text {cov }}$ or $\mathrm{NCE}_{\text {Zeff }}$ images and $\mathrm{N}_{\mathrm{CE}}$ is the total number of plaques detected on $\mathrm{CE}$ images.

\section{Statistical analysis}

Statistical analysis was performed using the statistical $\mathrm{R}$ software v 3.5 .3 (www.r-project.org/) and MedCalc v. 19 (MedCalc Software Ltd., Ostend, Belgium). Quantitative data were presented as mean \pm SD. The Shapiro-Wilk test was performed to assess normality of distribution. For 2 -group comparisons, an independent or paired $t$-test was performed for normally distributed data; and the MannWhitney or Wilcoxon signed-rank test was performed for non-normally distributed data, as appropriate. For $3+$ groups paired comparisons, a repeated analysis of variance (ANOVA) or Friedman test was performed, as appropriate. Plaque and vessel ROI areas were compared using the Bland-Altman plot to test the agreement between CE conventional images and Zeff images. Inter-reviewer agreement was evaluated by the intraclass correlation coefficient (ICC). The ICC values were interpreted as proposed by Koo and Li (21): below 0.50, poor; between 0.50 and 0.75 , moderate; between 0.75 and 0.90 , good; above 0.90 , excellent. A $\mathrm{P}$ value $<0.05$ was considered statistically significant.

\section{Results}

A total of 37 patients ( 25 men and 12 women) were enrolled in this study. The cases were $69.4 \pm 8.4$ years old, ranging from 50 to 82 years. A total of 86 atherosclerotic plaques confirmed by $\mathrm{CE}$ images were included in this study; they were distributed in the thoracic arteries (31/86 plaques, $36.0 \%$ ), abdominal arteries (42/86, 48.8\%), and carotid 
arteries $(13 / 86,15.1 \%)$. The demographic features of the patients and the distribution of the atherosclerotic plaques are summarized in Table 1.

The mean vessel ROI area derived from $\mathrm{CE}_{\mathrm{cov}}$ had no significant difference compared with that obtained from $\mathrm{CE}_{\text {Zeff }}\left(502.19 \pm 378.68\right.$ vs. $\left.498.14 \pm 371.54 \mathrm{~mm}^{2} ; \mathrm{P}=0.28\right)$. The Bland-Altman plot (Figure 1A) showed a bias of $4.05 \mathrm{~mm}^{2}$ [95\% confidence interval (CI): -1.53 to 9.63; upper limit of agreement (ULOA), 55.06; lower limit of agreement (LLOA), -46.96]. The agreement of both methods was excellent (ICC $=0.99$ ). The mean plaque ROI area obtained from $\mathrm{CE}_{\mathrm{cov}}$ was larger than that derived from $\mathrm{CE}_{\text {Zeff }}\left(81.45 \pm 35.55\right.$ vs. $\left.75.46 \pm 38.90 \mathrm{~mm}^{2} ; \mathrm{P}<0.05\right)$. The Bland-Altman plot (Figure 1B) showed a bias of $5.99 \mathrm{~mm}^{2}$ (95\% CI: 3.45 to 8.33; ULOA, 29.17; LLOA, -17.19); the agreement of both methods was excellent (ICC $=0.94)$. The plaque proportion on $\mathrm{CE}_{\mathrm{cov}}$ images was larger than that of the $\mathrm{CE}_{\text {Zeff }}$ data $(21 \%$ vs. $19 \% ; \mathrm{P}<0.05)$.

The detection rate of non-calcified plaques on

Table 1 Demographic data and distribution of atherosclerotic plaques

\begin{tabular}{lc}
\hline Variable & Total \\
\hline No. of patients & 37 \\
Age (years) & $69.4(50,82)$ \\
Men/women & $25(67.6 \%) / 12(32.4 \%)$ \\
No. of plaques & 86 \\
Thoracic artery $^{*}$ & 31 \\
Abdominal artery & 42 \\
Carotid artery & 11 \\
\hline
\end{tabular}

${ }^{*}$, data are mean and range; ${ }^{* \star}$, plaque distribution.
$\mathrm{NCE}_{\text {cov }}$ was $8.1 \%(7 / 86)$, while on $\mathrm{NCE}_{\text {Zeff }}$ images it was $54.7 \%$ (47/86). All 7 plaques detected by $\mathrm{NCE}_{\text {cov }}$ were also detected by $\mathrm{NCE}_{\text {Zeff }}$ For the 7 plaques detected both by $\mathrm{NCE}_{\text {cov }}$ and $\mathrm{NCE}_{\text {Zeff }}$, the CT attenuation and Zeff values of blood were both larger than those values of the plaques on NCE images $(42.00 \pm 5.23$ vs. 25.67 $\pm 14.80 \mathrm{HU}$; $7.33 \pm 0.08$ vs. $7.19 \pm 0.11 \mathrm{HU}$; both $\mathrm{P}<0.05$ ). There were no significant differences in the vessel ROI areas measured by $\mathrm{CE}_{\text {cov }}, \mathrm{CE}_{\text {Zeff }}$, and $\mathrm{NCE}_{\text {Zeff, }}$ which were all larger than that measured by $\mathrm{NCE}_{\text {cov }}(449.55 \pm 173.78$, $435.62 \pm 157.94,445.80 \pm 164.76$ vs. $328.10 \pm 101.87 \mathrm{~mm}^{2}$ ). There was no significant difference between the ROI areas of plaque measured by $\mathrm{CE}_{\text {cov }}$ and $\mathrm{CE}_{\text {Zeff, which were }}$ both larger than that measured by $\mathrm{NCE}_{\text {cov }}$ and $\mathrm{NCE}_{\text {Zeff }}$ $\left(77.76 \pm 23.41 \mathrm{~mm}^{2}, 62.80 \pm 14.71 \mathrm{~mm}^{2}\right.$ vs. $18.39 \pm 13.04 \mathrm{~mm}^{2}$, $48.73 \pm 20.28 \mathrm{~mm}^{2}$; all $\left.\mathrm{P}<0.05\right)$; the ROI area measured by $\mathrm{NCE}_{\text {cov }}$ was smaller than that measured by $\mathrm{NCE}_{\text {Zeff }}(\mathrm{P}<0.05)$. For the 47 plaques detected by $\mathrm{NCE}_{\text {Zeff }}$, the $\mathrm{CT}$ attenuation and Zeff values of blood and plaques on the NCE images showed no significant differences $(42.53 \pm 28.57 \mathrm{vs}$. $35.14 \pm 26.25$ HU; $\mathrm{P}=0.18 ; 7.32 \pm 0.23$ vs. $7.31 \pm 0.10 ; \mathrm{P}=0.71$ ). The CNR of Zeff was significantly higher than that of CT attenuation on the NCE images (1.69 vs. $1.12 ; \mathrm{P}<0.01)$. The ROI area of vessel measured by $\mathrm{NCE}_{\text {Zeff }}$ was larger than that measured by $\mathrm{CE}_{\text {Zeff }}(507.16 \pm 250.53$ vs. $489.52 \pm 241.27$ $\left.\mathrm{mm}^{2} ; \mathrm{P}<0.05\right)$. The ROI area of plaque measured by the $\mathrm{CE}_{\text {cov }}$ was larger than that measured by $\mathrm{CE}_{\text {Zeff }}$ and $\mathrm{NCE}_{\text {Zeff }}$ (84.68 \pm 37.62 vs. $79.76 \pm 39.82$ and $45.62 \pm 25.23 \mathrm{~mm}^{2}$; both $\mathrm{P}<0.05)$, and the ROI area measured by $\mathrm{CE}_{\text {Zeff }}$ was larger than that measured by $\mathrm{NCE}_{\text {Zeff }}(\mathrm{P}<0.05)$. More detailed results of plaque and vessel ROI areas are listed in Table 2. The Zeff value and ROI area (detected by Zeff mapping) of the plaques detected from both $\mathrm{NCE}_{\text {cov }}$ and $\mathrm{NCE}_{\text {Zeff }}$ showed no significant difference to those detected only by $\mathrm{NCE}_{\text {Zeff }}(7.19 \pm 0.12$ vs. $7.32 \pm 0.23 ; \mathrm{P}=0.10 ; 48.73 \pm 20.28$ vs.
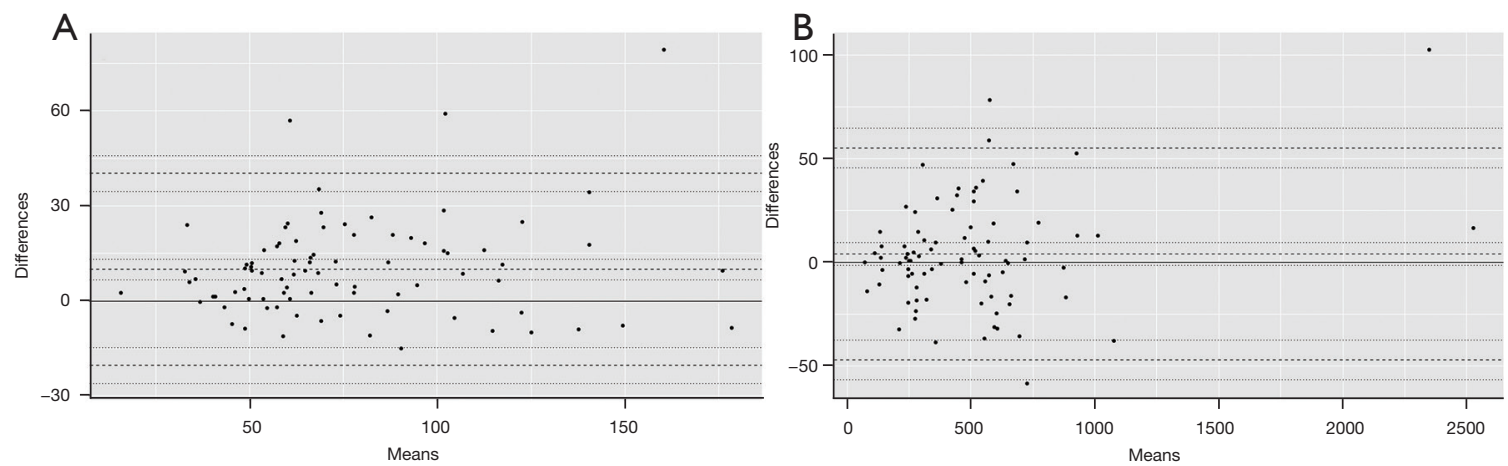

Figure 1 Bland-Altman plots showing agreement for area measurements between conventional and Zeff map images. (A) Plaque. (B) Vessel. 
Table 2 Plaque and vessel areas comparison among different image series

\begin{tabular}{|c|c|c|c|c|}
\hline & \multicolumn{2}{|c|}{ Plaques detected by $\mathrm{NCE}_{\text {cov }} \& \mathrm{NCE}_{\text {zeff }}$} & \multicolumn{2}{|c|}{ Plaques detected by $\mathrm{NCE}_{\text {Zeff }}$} \\
\hline $\mathrm{CE}_{\mathrm{cov}}$ & $77.76(23.41)^{\mathrm{b}, \mathrm{c}}$ & $449.55(173.78)^{b}$ & $84.68(37.62)^{\mathrm{a}, \mathrm{c}}$ & $494.61(240.96)$ \\
\hline $\mathrm{CE}_{\text {Zeff }}$ & $62.8(14.71)^{\mathrm{d}, \mathrm{e}}$ & $435.62(157.94)^{d}$ & $79.76(39.82)^{a, e}$ & $489.52(241.27)^{\mathrm{e}}$ \\
\hline $\mathrm{NCE}_{\text {cov }}$ & $18.39(13.04)^{\mathrm{b}, \mathrm{d}, \mathrm{f}}$ & $328.10(101.87)^{\mathrm{b}, \mathrm{d}, \mathrm{f}}$ & - & - \\
\hline F value & 20.25 & 5.85 & 75.26 & 3.52 \\
\hline$P$ value & $<0.01$ & $<0.01$ & $<0.01$ & 0.03 \\
\hline
\end{tabular}

Friedman test, a $P$ value of less than 0.05 was considered statistically significant. CE, contrast enhanced images; NCE, non-contrast enhanced images that could detect the plaques. Have significant difference after multiple comparisons: a: $C E_{\text {cov }}$ vs. $C E_{\text {Zeff; }}$ b: $C E_{\text {cov }}$ vs. $\mathrm{NCE}_{\text {cov }} ; \mathrm{c}: \mathrm{CE}_{\text {cov }}$ vs. $\mathrm{NCE}_{\text {Zeff }} ; \mathrm{d}: \mathrm{CE}_{\text {Zeff }}$ vs. $\mathrm{NCE}_{\text {cov }} ;$ e: $\mathrm{CE}_{\text {Zeff }}$ vs. $\mathrm{NCE}_{\text {Zeff }} ; \mathrm{f}: \mathrm{NCE}_{\text {cov }}$ vs. $\mathrm{NCE}_{\text {Zeff }}$.

45.08 $\pm 26.18 ; \mathrm{P}=0.73)$. There was no significant difference between the Zeff value of plaques detected by both $\mathrm{NCE}_{\text {Zeff }}$ and $\mathrm{NCE}_{\text {cov }}$ and those detected only by $\mathrm{NCE}_{\text {Zeff }}(7.19 \pm 0.12$ vs. 7.34 $\pm 0.24 ; \mathrm{P}=0.10)$. There was no significant difference between the Zeff value of plaques detected by both $\mathrm{NCE}_{\text {Zeff }}$ and $\mathrm{NCE}_{\text {cov }}$ and those detected only by $\mathrm{NCE}_{\text {Zeff }}(7.19 \pm 0.12$ vs. 7.34 $\pm 0.24 ; \mathrm{P}=0.10)$. The $\mathrm{Zeff}$ value of plaques detected by both $\mathrm{NCE}_{\text {Zeff }}$ and $\mathrm{NCE}_{\text {cov }}$ showed no significant difference to the value of those detected only by $\mathrm{NCE}_{\text {Zeff }}$ (7.19 \pm 0.12 vs. $7.34 \pm 0.24 ; \mathrm{P}=0.10)$. Inter-reviewer agreement was good $(\mathrm{ICC}=0.78)$.

\section{Discussion}

This study revealed that non-calcified atherosclerotic plaques could be detected on NCECT scans by reviewing Zeff map images generated from DLCT data. Compared with conventional CT, on NCECT scans, Zeff was more sensitive for non-calcified plaque detection (detection rate of $8.1 \%$ vs. $54.7 \%$ ).

Atherosclerosis is a systemic disease that leads to raised plaques within the vessel walls of arteries, such as the aorta, coronary artery, and carotid artery. Knowledge of a patient's atherosclerotic load is potentially clinically important. Atherosclerotic disease in the thoracic aorta has been linked to an increased risk of thromboembolic events, mortality, and stroke (22). Pathologically, atherosclerosis is a chronic inflammatory disease characterized by intimal thickening and plaque formation in the arteries (1). It is believed that a soft plaque forms first and is then calcified much later (23). Thus, detection of non-calcified plaques is challenging using NCECT scans, and a CECT scan is usually recommended for plaque-burden assessment. Therefore, it is important to identify the non-calcified plaques using NCECT imaging to avoid further CECT scans or other follow-up tests. A previous study (17) of conventional images indicated that noncalcified plaques could be visually detected by calcium scoring assays. The 2 observers identified 21 and 17 cases from 116 patients, respectively, with 38 and 35 suspected lesions, respectively. Some 33 of 38 (86.8\%; observer 1) and 31 of 35 (88.6\%; observer 2) lesions were confirmed by coronary CT angiography. Unlike the method in the above report, in this study, both reviewers were asked to identify non-calcified plaques on NCECT images in 37 patients with 86 lesions confirmed by CECT scans. Finally, 47 of the 86 (54.7\%) lesions were identified using Zeff map images and 7 plaques $(8.1 \%)$ were identified using conventional images derived from NCECT scans.

Although the Zeff value could be calculated by employing 2 different X-ray tube voltages in the early days of CT (24), it has clinical potential for lesion detection and material decomposition, since the DECT approach has become commercially available in recent years $(20,25,26)$. Unlike the tube-based DECT method, DLCT is a detector-based DECT solution, which does not require upfront decisionmaking or additional radiation doses. The excellent concordance between Zeff map and conventional CT image measurements of the mean vessel ROI area in this study showed that the Zeff can be another reliable quantitative indicator in CT imaging (Figure 2). A phantom study by Hua et al. (11) using DLCT showed median Zeff deviation ranging from $2.3 \%$ to $1.7 \%$ for soft tissue and bone inserts, which was more accurate than rapid-kVp-switching DECT (with an accuracy of 15\%) (27) or dual-source DECT (with 

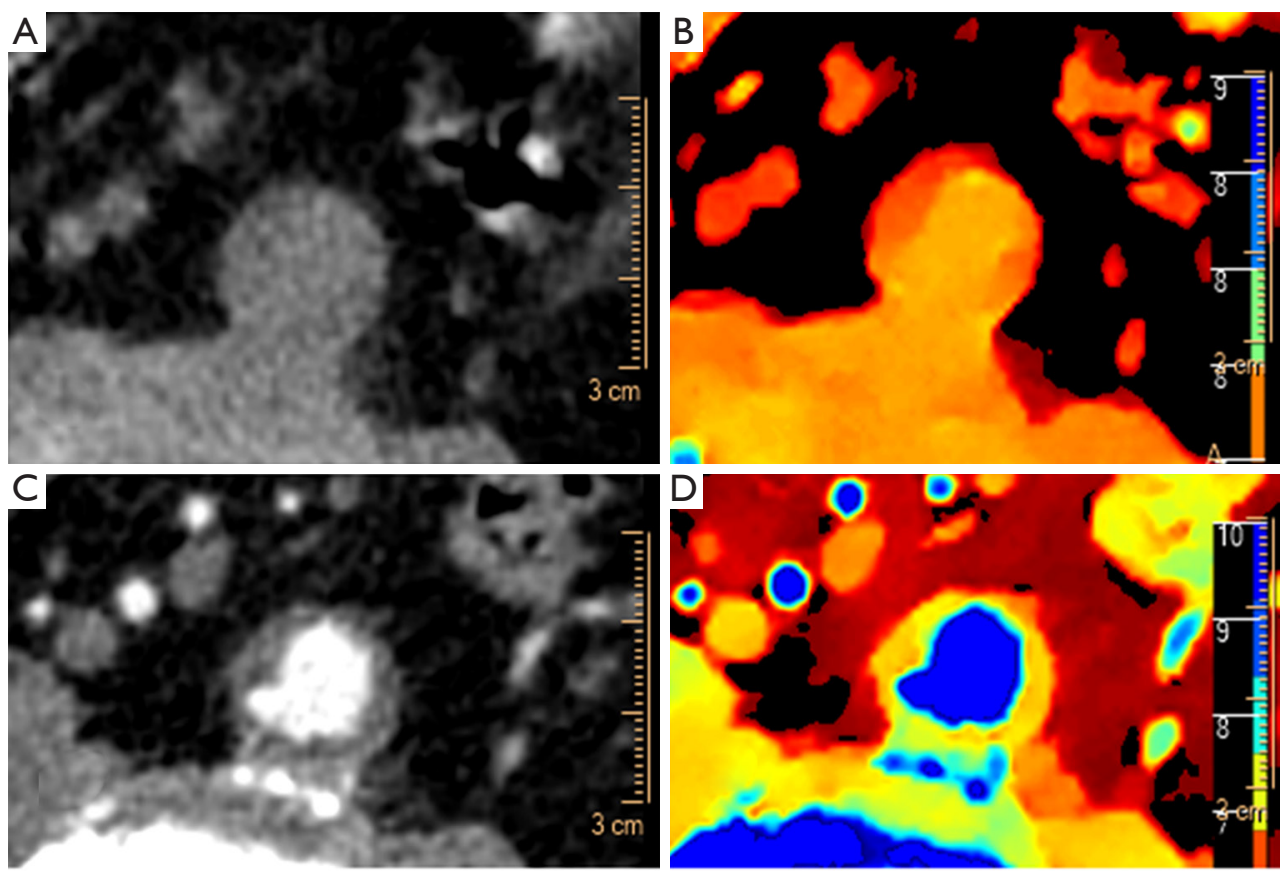

Figure 2 A non-calcified plaque in the abdominal artery. (A) Conventional image based on non-contrast CT scan. (B) Zeff image based on noncontrast-enhanced CT scan. (C) Conventional image based on contrast-enhanced scan. (D) Zeff image based on contrast-enhanced CT scan.

an accuracy of $6.2 \%$ ) (28). Moreover, this detector-based DECT solution also enabled noise suppression between the 2 layers, resulting in a higher CNR of the Zeff (1.69) in comparison with conventional CT attenuation (1.12) in this study. Thus, Zeff map images obtained from DLCT are sensitive for lesion detection, providing feasibility for their use in the identification of non-calcified plaques using NCECT scans.

This study had some limitations. Firstly, it did not evaluate the overall sensitivity and specificity of Zeff map images derived from NCECT scans for the identification of non-calcified plaques. Larger, population-based studies are warranted to assess atherosclerotic plaque burden using NCE spectral images. Secondly, because there is no available database of Zeff values for soft tissue and noncalcified plaques, image window width and center for Zeff mapping were not standardized, which might have introduced some measurement errors. Thirdly, fibrous and lipid rich plaques were not determined in this study.

In conclusion, this study showed that non-calcified atherosclerotic plaques could be identified on Zeff map images derived from NCECT scans using DLCT. This finding shall be useful for the screening of asymptomatic atrisk patients.

\section{Acknowledgments}

Funding: This work was supported by the Nanjing Medical Science and Technique Development Foundation (No. ZKX19018; No. QRX17057), the China Postdoctoral Science Foundation (No. 2019M661804), and the Jiangsu Province Postdoctoral Science Foundation (No. 2019k060).

\section{Footnote}

Reporting Checklist: The authors have completed the MDAR checklist. Available at https://qims.amegroups.com/article/ view/10.21037/qims-21-643/rc

Conflicts of Interest: All authors have completed the ICMJE uniform disclosure form (available at https://qims. amegroups.com/article/view/10.21037/qims-21-643/coif). $\mathrm{XC}$ and ZS serve as employees of the Philips Healthcare company. The other authors have no conflicts of interest to declare.

Ethical Statement: The authors are accountable for all aspects of the work and ensuring that questions related to the accuracy or integrity of any part of the work are 
appropriately investigated and resolved. The study was conducted in accordance with the Declaration of Helsinki (as revised in 2013). The study was approved by the Ethics Committee of the Nanjing Drum Tower Hospital, the Affiliated Hospital of Nanjing University Medical School (project ID: 2021-026-02), and individual consent for this retrospective analysis was waived.

Open Access Statement: This is an Open Access article distributed in accordance with the Creative Commons Attribution-NonCommercial-NoDerivs 4.0 International License (CC BY-NC-ND 4.0), which permits the noncommercial replication and distribution of the article with the strict proviso that no changes or edits are made and the original work is properly cited (including links to both the formal publication through the relevant DOI and the license). See: https://creativecommons.org/licenses/by-nc-nd/4.0/.

\section{References}

1. Mann DL, Zipes DP, Libby P, Bonow RO, Braunwald E. Braunwald's heart disease: a textbook of cardiovascular medicine. Philadelphia, PA: Elsevier/Saunders; 2015.

2. Hetterich H, Jaber A, Gehring M, et al. Coronary computed tomography angiography based assessment of endothelial shear stress and its association with atherosclerotic plaque distribution in-vivo. PLoS One 2015;10:e0115408.

3. Kolossváry M, Szilveszter B, Merkely B, et al. Plaque imaging with CT-a comprehensive review on coronary CT angiography based risk assessment. Cardiovasc Diagn Ther 2017;7:489-506.

4. Liu X, Yu L, Primak AN, et al. Quantitative imaging of element composition and mass fraction using dualenergy CT: three-material decomposition. Med Phys 2009;36:1602-9.

5. Große Hokamp N, Salem J, Hesse A, et al. Low-Dose Characterization of Kidney Stones Using Spectral Detector Computed Tomography: An Ex Vivo Study. Invest Radiol 2018;53:457-62.

6. McCollough CH, Leng S, Yu L, et al. Dual- and MultiEnergy CT: Principles, Technical Approaches, and Clinical Applications. Radiology 2015;276:637-53.

7. Kalisz K, Halliburton S, Abbara S, et al. Update on Cardiovascular Applications of Multienergy CT. Radiographics 2017;37:1955-74.

8. Große Hokamp N, Maintz D, Shapira N, et al. Technical background of a novel detector-based approach to dual- energy computed tomography. Diagn Interv Radiol 2020;26:68-71.

9. Huang X, Gao S, Ma Y, et al. The optimal monoenergetic spectral image level of coronary computed tomography (CT) angiography on a dual-layer spectral detector CT with half-dose contrast media. Quant Imaging Med Surg 2020;10:592-603.

10. Maass C, Baer M, Kachelriess M. Image-based dual energy CT using optimized precorrection functions: a practical new approach of material decomposition in image domain. Med Phys 2009;36:3818-29.

11. Hua CH, Shapira N, Merchant TE, et al. Accuracy of electron density, effective atomic number, and iodine concentration determination with a dual-layer dualenergy computed tomography system. Med Phys 2018;45:2486-97.

12. Ehn S, Sellerer T, Muenzel D, et al. Assessment of quantification accuracy and image quality of a full-body dual-layer spectral CT system. J Appl Clin Med Phys 2018;19:204-17.

13. Rajiah P, Rong R, Martinez-Rios C, et al. Benefit and clinical significance of retrospectively obtained spectral data with a novel detector-based spectral computed tomography - Initial experiences and results. Clin Imaging 2018;49:65-72.

14. Sun J, Zhang Z, Lu B, et al. Identification and quantification of coronary atherosclerotic plaques: a comparison of 64-MDCT and intravascular ultrasound. AJR Am J Roentgenol 2008;190:748-54.

15. Qanadli SD, Jouannic AM, Dehmeshki J, et al. CT attenuation values of blood and myocardium: rationale for accurate coronary artery calcifications detection with multi-detector CT. PLoS One 2015;10:e0124175.

16. Dey D, Callister T, Slomka P, et al. Computeraided detection and evaluation of lipid-rich plaque on noncontrast cardiac CT. AJR Am J Roentgenol 2006;186:S407-13.

17. Thilo C, Gebregziabher M, Mayer FB, et al. Can noncalcified coronary artery plaques be detected on noncontrast CT calcium scoring studies? Acad Radiol 2011;18:858-65.

18. Oda S, Nakaura T, Utsunomiya D, et al. Clinical potential of retrospective on-demand spectral analysis using duallayer spectral detector-computed tomography in ischemia complicating small-bowel obstruction. Emerg Radiol 2017;24:431-4.

19. Saito H, Noda K, Ogasawara K, et al. Usefulness and limitations of dual-layer spectral detector computed 
tomography for diagnosing biliary stones not detected by conventional computed tomography: a report of three cases. Clin J Gastroenterol 2018;11:172-7.

20. Taguchi N, Oda S, Kobayashi T, et al. Advanced parametric imaging for evaluation of Crohn's disease using dual-energy computed tomography enterography. Radiol Case Rep 2018;13:709-12.

21. Koo TK, Li MY. A Guideline of Selecting and Reporting Intraclass Correlation Coefficients for Reliability Research. J Chiropr Med 2016;15:155-63.

22. Russo C, Jin Z, Rundek T, et al. Atherosclerotic disease of the proximal aorta and the risk of vascular events in a population-based cohort: the Aortic Plaques and Risk of Ischemic Stroke (APRIS) study. Stroke 2009;40:2313-8.

23. Cho I, Suh JW, Chang HJ, et al. Prevalence and prognostic implication of non-calcified plaque in asymptomatic population with coronary artery calcium score of zero. Korean Circ J 2013;43:154-60.

24. Rutherford RA, Pullan BR, Isherwood I. Measurement

Cite this article as: Chen W, Li R, Yin K, Liang J, Li H, Chen X, Sheng Z, Yu H, Mu D. Clinical feasibility of using effective atomic number maps derived from non-contrast spectral computed tomography to identify non-calcified atherosclerotic plaques: a preliminary study. Quant Imaging Med Surg 2022;12(4):2280-2287. doi: 10.21037/qims-21-643 of effective atomic number and electron density using an EMI scanner. Neuroradiology 1976;11:15-21.

25. Mandal SR, Bharati A, Haghighi RR, et al. Non-invasive characterization of coronary artery atherosclerotic plaque using dual energy CT: Explanation in ex-vivo samples. Phys Med 2018;45:52-8.

26. Haghighi RR, Chatterjee S, Tabin M, et al. DECT evaluation of noncalcified coronary artery plaque. Med Phys 2015;42:5945-54.

27. Goodsitt MM, Christodoulou EG, Larson SC. Accuracies of the synthesized monochromatic CT numbers and effective atomic numbers obtained with a rapid $\mathrm{kVp}$ switching dual energy CT scanner. Med Phys 2011;38:2222-32.

28. Almeida IP, Schyns LE, Öllers MC, et al. Dual-energy CT quantitative imaging: a comparison study between twin-beam and dual-source CT scanners. Med Phys 2017;44:171-9. 